Revue

de Sémantique

et Pragmatique
Revue de Sémantique et Pragmatique

39 | 2016

Varia

\title{
Humour et métaphore : quelques éléments d'une analogie pour la construction d'un sens inattendu
}

Illustration sur un corpus de citations de George Bernard Shaw

Pierre-Yves Raccah

\section{OpenEdition}

\section{Journals}

Édition électronique

URL : http://journals.openedition.org/rsp/420

DOI : $10.4000 /$ rsp. 420

ISSN : 2610-4377

\section{Éditeur}

Presses universitaires d'Orléans

Édition imprimée

Date de publication : 1 octobre 2016

Pagination : 75-94

ISSN : 1285-4093

\section{Référence électronique}

Pierre-Yves Raccah, «Humour et métaphore : quelques éléments d'une analogie pour la construction d'un sens inattendu », Revue de Sémantique et Pragmatique [En ligne], 39 | 2016, mis en ligne le 01 octobre 2017, consulté le 01 mai 2019. URL : http://journals.openedition.org/rsp/420 ; DOI : 10.4000/ rsp. 420 


\title{
HuMOUR ET MÉTAPHORE : QUELQUES ÉLÉMENTS D'UNE ANALOGIE POUR LA CONSTRUCTION D'UN SENS INATTENDU. ILLUSTRATION SUR UN CORPUS DE CITATIONS DE George Bernard Shaw
}

\author{
Pierre-Yves Raccah, \\ CNRS, UMR 7270 - LLL - pyr@linguistes.fr
}

\section{INTRODUCTION}

Lorsqu'on essaye de comprendre pourquoi un bon mot nous a fait rire, on a souvent à l'esprit une sensation de piège dans lequel on est tombé : c'est la chute du bon mot qui nous fait ... tomber dans ce piège. Et le trait est d'autant plus drôle que le piège était inattendu et inévitable. Cette caractéristique, que nous nous efforçons de préciser et de formuler de manière plus théorique, s'apparente à ce que l'on peut dire d'une métaphore intéressante : inattendue et inévitable (après coup...). Nous montrons que ces 'impressions' ne sont pas nécessairement aussi vagues et insaisissables qu'elles pourraient sembler : une fois précisées, elles pourront constituer des caractéristiques d'un fait de langue qui concerne et les bons mots et les métaphores intéressantes...

La première section est une analyse informelle, une sorte de commentaire de texte, destiné à problématiser les questions, concernant l'humour, que nous aborderons, à propos d'un choix de «bons mots » de George Bernard Shaw. Dans la section 2, nous proposons un modèle sémantique partiel, descriptif des phénomènes liés à l'humour, en nous appuyant sur ces citations de GBS, et en utilisant quelques unes des réflexions théoriques de Attardo (1994). Nous mon- 
trons ensuite que ce modèle partiel est suffisamment général pour s'appliquer à d'autres énoncés humoristiques, notamment aux autres citations du corpus, ce qui légitime l'idée selon laquelle les outils descriptifs ainsi construits permettent effectivement des descriptions de propriétés des langues et pas seulement des discours. Chemin faisant, nous verrons que la simple reconnaissance des faits que nous avons mis en évidence (comme, par exemple, l'existence de deux phases de constructions du sens, avec des résultats incompa-tibles) disqualifie la conception 'classique' de la communication langagière, selon laquelle les langues servent à coder et à décoder un sens pour qu'un locuteur puisse le transmettre au moyen d'un discours. On verra, toujours dans la section 2, qu'une prise en compte de ces faits appelle, une conception 'manipulatoire' de la communication langagière, selon laquelle une langue constitue une boîte à outils permettant au locuteur qui l'utilise de faire construire, par un interlocuteur, un sens proche de ce qu'il (le locuteur) veut qu'il (l'interlocuteur) construise.

Dans la troisième section, nous appliquons ces outils à la description sémantique de l'effet métaphorique : nous n'apportons pas une contribution originale à la caractérisation rhétorique de la métaphore ; d'ailleurs, nous nous intéresserons surtout aux métaphores nominales, et en insistant sur leur aspect sémantique, nous verrons, ce faisant, que la notion de métaphore ne peut pas constituer un concept linguistique, car elle introduirait un paradoxe qui interdirait à toute métaphore de produire un effet métaphorique... Nous verrons que l'effet métaphorique est un cas particulier de l'effet de sens général : cette remarque nous permettra de plonger le modèle partiel pour la description de l'effet métaphorique dans un modèle sémantique plus général. Nous concluons cette étude, d'une part, en justifiant l'idée que les propriétés sémantiques d'un 'bon' mot d'esprit sont analogues à celles d'une 'bonne' métaphore, accréditant ainsi que l'humour et la métaphore relèvent de faits de langue simiᄀlaires et, d'autre part, sur un plan plus méthodologique, en montrant en quoi les différents as $\neg$ pects abordés concourent à suggérer qu'une bonne théorie de la signification ne peut consiᄀdérer ni le jeu de mot ni la métaphore comme des exceptions, mais doit, au contraire, être en mesure de les décrire avec les mêmes outils que ceux qu'elle utilise pour rendre compte des phénomènes sémantiques «normaux ».

\section{REMARQUES PRÉLIMINAIRES SUR QUELQUES EXEMPLES EXTRAITS DU CORPUS}

Cette section vise principalement à délimiter le genre de questions sur l'humour auquel ce travail tente de fournir des réponses partielles. Les commentaires non théorisés de quelques exemples tirés du corpus de 'bons mots' de GBS nous permettront, par abduction, de faire quelques hypothèses sur les mécanismes de l'humour, hypothèses que nous préciserons, et tenterons de généraliser, dans la section suivante, avant de comparer cette généralisation à 
celle qui préside aux métaphores. Le corpus choisi est un corpus heuristique et non un corpus de preuve : il ne sert qu'à alimenter le raisonnement abductif qui fournira nos hypothèses théoriques. Un travail expérimental ultérieur, visant à valider le modèle descriptif proposé, devra, s'il est jugé utile, s'appuyer sur un corpus plus vaste, plus varié, mieux spécifié, et contenant des indices des processus d'interprétation.

\section{Exemple 1 Isadora}

Isadora Duncan aurait un jour dit à George Bernard Shaw ${ }^{1}$ :

"We should have a child together. Think of it! With my body and your brains, what a wonder it would be."

Mais Shaw lui aurait répondu :

"Yes. But what a tragedy if it had my body and your brains?"

Le sel de cette réponse de Shaw est que, tout en ne contestant ni le raisonnement eugénique d'Isadora, ni sa conviction d'être, elle, très belle, ni sa conviction que lui est intelligent, cette réponse permet Shaw d'insulter les capacités intellectuelles d'Isadora en laissant entendre que c'est lui qui est laid. La « tragedy » provient de ce que les enfants peuvent être le produit de ce qu'il y a de meilleur chez leurs parents, mais aussi de ce qu'il y a de pire... Au couple $<$ beauté,intelligence $>$ que Duncan présente comme étant celui qu'elle forme avec Shaw, ce dernier, par sa réponse, oppose le couple <bêtise,laideur>; et pour qu'il appelle cela une «tragedy », il faut vraiment que la bêtise soit profonde!

\section{Exemple 2 Democracy}

"Democracy is a device that ensures we shall be governed no better than we deserve."

Cette opinion, qui se présente au début comme invoquant une opinion positive sur la démocratie, se termine comme un jugement sévère sur l'humanité, sauf si l'on est prêt à revenir sur les a priori positifs concernant la démocratie. En effet, si l'on trouve 'bonne' la démocratie, en acceptant l'opinion de Shaw, on doit aussi trouver 'bon' que l'humanité soit punie de son démérite, justement par la démocratie : ce paradoxe n'est évité que si l'on renonce à cette première opinion sur la démocratie.

\section{Exemple 3 Criminals}

"Criminals do not die by the hands of the law. They die by the hands of other men."

En rappelant que ce sont les êtres humains qui exécutent les décisions de justice (double sens de « hands »), cette 'sentence' constitue un argument logique contre la peine de mort en soulignant le paradoxe suivant : s'il est crimi-

${ }^{1}$ Il existe de nombreuses versions de cette anecdote, toutes faisant intervenir le même raisonnement et son renversement. 
nel de tuer des êtres humains, et puisque la peine de mort est administrée par des êtres humains sur d'autres êtres humains, cette peine crée des criminels. Avant de lire la seconde phrase, le lecteur à qui Shaw se présente comme s'adressant a une conception sociétale de ce qu'est un « criminal » (conception renforcée par la métaphore " hands of law») ; la seconde phrase l'oblige à revoir cette conception («other men »), et c'est cette révision qui va le pousser à conclure que les bourreaux sont, eux aussi, des « criminals».

\section{Exemple 4 Hell}

"Hell is full of musical amateurs."

Cette formule joue sur la double fonction reconnue à l'enfer : (a) une punition et (b) un lieu où on est puni. Ainsi, elle permet de dire, en même temps, et que les musiciens amateurs constituent une punition et qu'ils doivent être punis (sans doute pour cette raison). L'effet humoristique vient de ce que, après avoir choisi l'une des fonctions de l'enfer pour interpréter le début de l'aphorisme (les X sont punis de l'enfer ou les X constituent l'une des punitions infligées en enfer), on se rend compte que, à cause de ce choix, l'autre fonction convient à l'interprétation. Dans cet exemple, il n'y a pas d'incompatibilité entre les faits, mais entre celui des deux points de vue sur l'enfer dont parle l'aphorisme.

\section{Exemple 5 England}

"England and America are two countries separated by a common language."

Cet aphorisme souligne l'absence de fondement de l'idée reçue selon laquelle ce qui est commun rapproche. La première réaction qu'il suscite est, en effet, de vérifier que l'on a bien lu « separated », et non pas, par exemple, « linked », « related » ou même « associated » : c'est seulement après avoir décelé la contradiction idéologique entre « separated» et « common», que l'on parvient à comprendre la critique de l'idée reçue : pour ce faire, il faut passer par la construction d'un autre sens, non idéologique, pour « common». Une étape supplémentaire, optionnelle celle-là, peut amener à attribuer à Shaw une critique de la bêtise humaine, qui fait que les peuples, au lieu de profiter de ce qu'ils ont en commun, s'en servent pour se quereller.

\section{Exemple 6 Everything}

"Everything happens to everybody sooner or later if there is time enough."

La compréhension de cette formule s'appuie très probablement sur un vaet-vient entre l'interprétation idiomatique de « sooner or later » (tôt ou tard, un jour ou l'autre) et sa 'dés-idiomatisation' partielle (« later »= plus tard) ${ }^{2}$.

${ }^{2}$ Deux arguments tendent à confirmer cette hypothèse : (i) une transformation de la formule en « Everything happens to everybody some day or other if there is time enough » enlèverait beaucoup de sel à l'aphorisme; (ii) la (mauvaise) traduction française standard : « tout arrive tôt ou tard à chacun s'il y a assez de temps » peut difficilement être considérée comme 
Ce subterfuge permet de contester l'idéologie selon laquelle les êtres humains seraient égaux devant une Fortune aveugle (donc, prétendue impartiale), et ce, en insistant sur l'inscription des vies humaines dans une temporalité finie.

\section{POURQUOI UN MÉCANISME SÉMANTIQUE POUR L'HUMOUR ?}

Nous allons, dans un premier temps, justifier l'idée que l'humour relève d'un mécanisme propre à la sémantique des langues humaines : pour ce faire, nous reviendrons sur une distinction déjà étudiée entre humour et comique ${ }^{3}$, et montrerons brièvement que les effets relevant du premier nécessitent le recours au langage et, en particulier, à l'interprétation. Par abstraction à partir des remarques concernant ces exemples, nous tentons ensuite de mettre en évidence un mécanisme général qui rende compte de l'effet humoristique des énoncés considérés comme des mots d'esprit ou des traits d'humour.

\subsection{HUMOUR VS. COMIQUE}

Il arrive que l'on rie d'une grimace, d'une facétie, d'une pitrerie ou d'une clownerie, produite volontairement ou non, par un être humain (appelons-le $\mathrm{C}$ ). Dans ces cas, même lorsque ce que $\mathrm{C}$ a produit est volontaire, on ne dirait pas que $\mathrm{C}$ a fait de l'humour. En fait, ce dont on rit, dans ces cas, c'est du comportement de $\mathrm{C}$ ou d'un personnage imaginaire que $\mathrm{C}$ incarne (ou 'joue').

Pour que l'on puisse parler d'humour à propos d'une production de $\mathrm{C}$, outre le résultat plus ou moins observable (rire ou sourire), trois conditions semblent nécessaires.

1. Pour que l'on puisse dire que $\mathrm{C}$ a fait de l'humour, il est nécessaire que $\mathrm{C}$ ait produit un énoncé. Cette contrainte permet d'exclure de l'humour le simple comique de position. Nous allons voir qu'elle n'est pas suffisante : deux conditions supplémentaires sont encore nécessaires.

2. Il est nécessaire que $\mathrm{C}$, en tenant les propos qu'il a tenu, ait eu l'intention de faire rire au moyen de ce qu'il s'apprêtait à dire. En effet, si les propos de $\mathrm{C}$ font rire alors que $\mathrm{C}$ n'avait aucune intention de provoquer l'hilarité, là non plus, on ne pourra pas considérer que $\mathrm{C}$ faisait de l'humour : on dira plutôt que l'on rit de lui, qu'il s'est ridiculisé.

un trait d'humour (sauf pour un lecteur qui sait qu'il s'agit d'une traduction de l'anglais et qui connaît bien l'expression idiomatique originale), ce qui n'est pas le cas pour la traduction légèrement modifiée : « tout arrive tôt ou tard à chacun s'il n'est pas trop tard ».

${ }^{3}$ Voir, par exemple, Alvarez (1982: 25) : «Ce qui m'intéresse [...] c'est de montrer qu'en utilisant les structures linguistiques pour faire de l'humour, ou en réagissant par le rire à un fait langagier donné, l'homme le plus « ordinaire», sans aucune connaissance explicite des règles qui régissent sa langue, révèle [un] savoir linguistique intériorisé que d'aucuns appellent «compétence». Si le rire peut nous mener vers les profondeurs de l'âme humaine, comme le voulait Freud, il peut aussi nous conduire vers la compétence linguistique [...]». 
Mais le fait de produire un énoncé en ayant l'intention de faire rire n'est pas encore suffisant pour que l'on parle d'humour, même si le public a effectivement ri.

3. Il est nécessaire que l'hilarité soit provoquée par le sens des propos de $\mathrm{C}$, tel que C l'a lui-même construit : en cas contraire, on est ramené au cas précédent, et l'on rit de $\mathrm{C}$ (ou du personnage auquel $\mathrm{C}$ confère la responsabilité des propos) et non pas de ce qu'il a dit.

L'humour est donc bien une question sémantique (et pas seulement pragmatique), puisqu'il suppose la production d'un énoncé et la construction d'un sens sur des bases qui soient communes au locuteur et à son public : la prise en compte de la seule situation n'est pas suffisante pour caractériser l'humour. Aussi bien Attardo (1994) que Alvarez (1982) l'avaient déjà signalé, mais n'en ont pas tiré les conséquences que nous en tirons au paragraphe suivant (ou, du moins, ne les ont pas formulées).

\subsection{UN MODĖLE SÉMANTIQUE DE L'EFFET HUMORISTIQUE}

Dans chacun des exemples précédents, nous avons mis en évidence une sorte d'ambiguïté, dans un sens très général (et encore un peu vague) du mot, dont les deux pôles, incompatibles entre eux ${ }^{4}$, sont l'un et l'autre, nécessaires à la compréhension de la formule.

Attardo (1994 : 95) formulait déjà une idée analogue à propos de phases et d'incompatibilité de sens :

\section{The Process of Disambiguation and the Joke}

A theory of the processing of the text of jokes must distinguish two moments in the disambiguation of a joke text [...]: in the first part of the process, a first isotopy / sense $\left(S_{1}\right)$ is established, until the recipient encounters an element that causes the passage from the first sense to a second sense $\left(S_{2}\right)$ antagonistic to the first one. The passage from $S_{1}$ to $S_{2}$ must be "unexpected", on the one hand, and "immediate" (i.e., should not involve "exceptional" mental expenditure), on the other.

Plus précisément, dans le corpus que nous avons partiellement analysé, le processus de construction du sens fait intervenir au moins deux phases, et le sens que l'on doit construire au terme de la dernière phase $\left(S_{f}\right)$ a au moins un aspect incompatible avec celui que l'on a construit à l'issue des phases précédentes $\left(\mathrm{S}_{\mathrm{i}}\right)$. Cette incompatibilité amène le lecteur à remettre en cause l'interprétation $S_{i}$; dans les meilleurs cas d'humour, cette remise en cause s'avère impossible : $\mathrm{S}_{\mathrm{f}} \mathrm{s}$ 'appuie sur les mêmes instructions sémantiques que celles qui ont conduit à $\mathrm{S}_{\mathrm{i}}$.

${ }^{4}$ Voir Lakof (1970) pour une brillante démonstration linguistique de l'incompatibilité des sens dus à une ambiguïté (contrairement à ceux dus à une faible spécification). 
On peut considérer que notre analyse ajoute aux suggestions de Attardo à la fois une touche de généralité (plusieurs phases y sont possibles au lieu des deux de Attardo) et une touche de précision (à la place des notions visées, chez Attardo, par "antagonistic", "unexpected", et "immediate"), nous faisons appel à des concepts pouvant s'affranchir d'un réductionnisme mental (incompatibilité, identité des instructions sémantiques), ce qui ne nous oblige plus à parler de «"exceptional" mental expenditure »

Comme nous l'avons vu au paragraphe précédent, pour qu'il y ait humour, il est nécessaire que le caractère incompatible des deux aspects du sens construit ne soit pas dû uniquement au monde objectif ou subjectif (la situation ou la manière selon laquelle le destinataire la voit) : l'incompatibilité doit provenir de la contribution sémantique des unités de langue utilisées, pour qu'elle soit bien perçue comme volontaire et non pas comme imputable à une erreur ou une incompétence du locuteur ${ }^{5}$. Un modèle de description sémantique est donc nécessaire (même s'il n'est sans doute pas suffisant) pour rendre compte des effets humoristique ; un tel modèle doit être en mesure de rendre compte des attentes que le sens construit génère sur les situations évoquées dans les énoncés, car c'est précisément la déception de ces attentes qui signale l'incompatibilité entre ce qui vient d'être construit et ce qui l'a été lors d'une phase antérieure ${ }^{6}$.

Pour le moment, le modèle partiel du mécanisme de l'humour (du moins dans les cas du genre de ceux de notre corpus et du genre de ceux qu'Attardo a étudiés) peut être représenté par le schéma suivant :

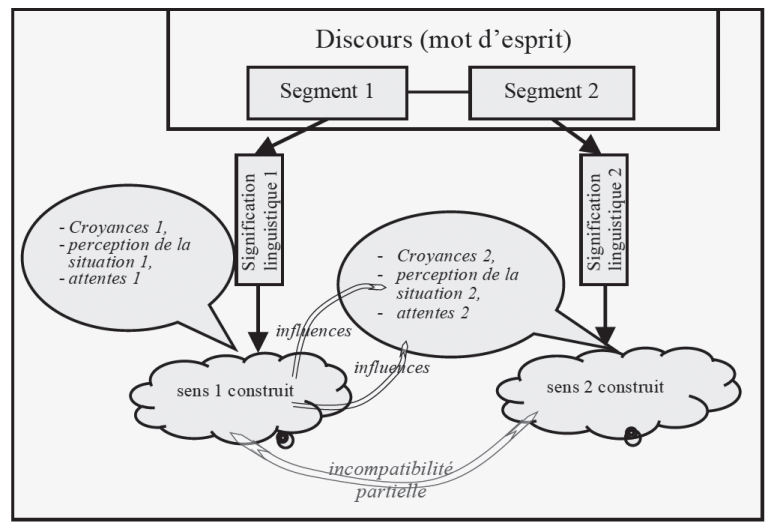

Schéma 1 : modèle partiel de l'effet humoristique

${ }^{5}$ Nous avons aussi vu que Alvarez (1994) soulignait déjà le caractère linguistique de l'humour; nous soulignons ici son aspect sémantique.

${ }^{6}$ C'est cette conséquence, que nous tirons de l'analyse précédente, qui différencie notre approche des autres conceptions fondées sur les mêmes observations. Nous verrons plus loin son intérêt fonctionnel, mais insistons tout de suite sur un avantage a priori : elle permet d'éviter l'alternative entre (i) réduire la sémantique à des phénomènes mentaux et (ii) exclure de la sémantique le mécanisme de l'humour. 
Comme l'indique le schéma 1, un premier segment du discours que le mot d'esprit constitue amène à construire un premier sens, en s'appuyant sur certaines croyances (ou connaissances), une certaine perception de la situation, et certaines attentes. Ce sens construit à l'issue du premier segment participe à la détermination d'un nouvel état de croyance, de perception de la situation et d'attentes, état dans lequel l'interprétation du second segment conduira à un sens (au moins partiellement) incompatible avec le premier.

\subsection{CONSÉQUENCES SUR LA CONFIGURATION DE LA SÉMANTIQUE ET DE LA COMMUNICATION}

Une caractéristique essentielle de ce modèle partiel de l'effet humoristique, nous l'avons vu, est qu'il fait intervenir au moins deux phases de construction du sens, correspondant aux segments consécutifs du discours, et qui soient telles que le sens construit au terme de la dernière phase soit, au moins partiellement, incompatible avec le sens construit au terme des phases précédentes, cette incompatibilité devant provenir de la signification elle-même et pas seulement des points de vue ou des croyances des interlocuteurs. Ce modèle partiel suppose donc que l'on puisse décrire les faits relatifs à un discours humoristique en termes de phases, de construction du sens, d'influence de la signification sur le sens construit, et d'influences réciproques du sens sur les points de vue et sur les attentes. Une telle conception est, on le voit sans difficulté, incompatible avec une conception de la communication dans laquelle le sens serait transmis par les discours : pour percevoir les effets des discours humoristiques, il est nécessaire (mais pas suffisant) de voir les discours comme suscitant la construction d'un sens (et non pas comme transmettant un sens). Si l'on admet que l'effet humoristique concerne bien, au moins partiellement, la sémantique, il faut alors renoncer à la conception habituelle (transmissionnelle) de la communication langagière, et lui substituer une conception que l'on pourrait appeler instructionnelle ou manipulatoire, selon laquelle les unités de langue donnent des instructions pour construire des sens, de sorte que les locuteurs profèrent des réalisations de segments linguistique dans le but de faire construire, par les destinataires, des sens proches de ceux que lesdits locuteurs voulaient que leurs destinataires construisissent. L'effet humoristique peut ainsi être conçu comme une volte-face délibérée dans la stratégie de manipulation.

En outre, puisque le second segment doit amener à construire un sens incompatible avec les attentes suscitées par la compréhension du premier segment, et/ou avec la perception de la situation influencée par la compréhension du premier segment, il est nécessaire que les attentes et les points de vue sur les situations puissent être exprimées dans le même formalisme que celui par lequel on exprime le sens construit. 
Les théories sémantiques vérifiant ces deux contraintes s'appuient donc sur une conception manipulatoire de la communication langagière, dans laquelle les unités de langue, simples ou complexes, utilisées dans un discours fournissent des instructions pour construire du sens à partir des points de vue admis préalablement à ce discours. Nous en reparlerons dans la section 4.

\section{MÉTAPHORE ET CONSTRUCTION DU SENS}

Une des plus belles caractérisations de l'effet métaphorique remonte au Moyen-Âge ; elle est due à un auteur franco-anglais, Geoffroi de Vinsauf':

Quando tuum proprium transsumis, plus sapit istud

Quod venit ex proprio. Talis transsumptio verbi

Est tibi pro speculo : qui ate specularis in illo

Et proprias cognoscis oves in rure alieno ${ }^{8}$

de Vinsauf : Poetria Nova (entre 1208 et 1214) in Faral 1924, vers 797 à 800

Cette caractérisation métaphorique de l'effet métaphorique est très suggestive mais ne dit rien de ce qui permet de produire de tels effets; un credo presque universel prétend néanmoins compenser cette absence, en se fondant sur ce qui est généralement présenté comme le bon sens élémentaire : nous ne contesterons pas le caractère élémentaire de ce credo, mais, sans avoir à étudier en détail les différents types de métaphores ${ }^{9}$ ni les différentes approches qui manifestent ce credo à des degrés divers ${ }^{10}$, nous montrerons que, loin de s'appuyer sur le bon sens, il va précisément dans le mauvais sens, en impliquant qu'une métaphore ne peut pas produire d'effet métaphorique... Nous proposons ensuite une autre conception de ce qui peut produire des effets métaphoriques, conception dont on verra qu'elle amène à considérer que les causes d'effets métaphoriques sont de même nature que ce qui fait construire du sens.

\footnotetext{
Salvo, etc.

8 "When you transpose a word whose literal meaning is proper to man, it affords greater pleasure, since it comes from what is your own. Such a metaphor serves you as a mirror, for you see yourself in it and recognize your own sheep in another's field". Traduction anglaise de Margaret Nims. La traduction française de la dernière ligne (celle qui nous intéresse le plus ici) est : «Et tu reconnais tes propres brebis dans le champ d'autrui ».

${ }^{9}$ On trouvera une telle étude dans Tamine (1979).

${ }^{10}$ Un tel travail serait très éloigné des objectifs de cet article ; il a d'ailleurs été brillamment réalisé par Paul Ricœur (1975), pour ce qui concerne ce qui a été écrit sur la métaphore depuis Aristote jusqu'à $1975 \ldots$
}

${ }^{7}$ Galfredus de Vino Salvo ou, selon les pays, Geoffrey of Vinsafe, Godefreddo di Vino 


\subsection{CE QUE LES MÉTAPHORES NE PEUVENT PAS ÊTRE}

a) Caractéristiques de la conception la plus répandue (conception « classique »)

On enseigne presque partout que :

Les métaphores modifient le sens des mots, transformant ainsi un 'sens propre' en 'sens figuré'.

Le lecteur averti, qui trouverait déjà cette conception « pléthonomique » inacceptable et douterait qu'une telle absurdité soit effectivement admise et enseignée, devrait, en principe, avoir raison... Mais malheureusement, les faits (et les textes) sont têtus (autant que les idéologies qui leur sont sous-jacentes) et l'optimiste de principe, à la lecture de la plupart des textes sur la métaphore, devra modérer sa confiance exagérée. À titre d'illustration, nous reprendrons, par ordre alphabétique des auteurs auxquels elles ont été attribuées, quelques unes des conceptions de la métaphore qui sont utilisées et enseignées. Cette brève analyse critique ne rend pas justice à la qualité des travaux de ces auteurs, mais ce n'est pas son objectif (ces auteurs n'ont d'ailleurs pas besoin qu'on leur rende hommage) : il s'agit de montrer qu'ils partent tous de cette conception de la métaphore dont je vais montrer qu'elle ne peut être qu'erronée, ou qu'on leur attribue cette conception.

Ainsi, Aristote, selon Marie-Christine Lala (2005 : 147) nous rappelle que :

La plupart du temps, le point de départ d'une étude de la métaphore s'ancre dans sa définition aristotélicienne, selon laquelle « la métaphore est le transport à une chose d'un nom qui en désigne une autre, transport ou du genre à l'espèce, ou de l'espèce au genre, ou de l'espèce à l'espèce ou d'après le rapport d'analogie ${ }^{11}$,

Néanmoins, Tamba et Veyne (1975 : 77-78) contestent l'interprétation, selon eux, anachronique, des textes d'Aristote, que cette attribution suppose :

Le malheur est qu'Aristote est antérieur de deux siècles au moins aux rhéteurs qui ont édifié la doctrine en question [celle du changement de sens] ; sa metaphora n'est pas notre métaphore, ce trope ou figure de signification qui consiste à changer le sens d'un mot en s'appuyant sur une ressemblance. [...] Il n'instaure pas davantage une sémantique des mots, fondée sur la discrimination d'un sens propre ou premier et d'un sens figuré ou détourné, qui serait second.

Barthes (S.E.L.F. 1966 : 78, reproduit dans (Barthes 1985), stipule que

Le [groupe des métaboles] comprend tous les connotateurs qui comportent une conversion sémantique ; soit la métaphore : voyageuse de nuit $=$ vieillesse $;$ la chaîne sémantique s'établit de la façon suivante : Sa1 (/voyageuse de nuit/) = Sé1 ("voyageuse de nuit") = Sé2 ("vieillesse") = Sa2 (/vieillesse/) ; dans cette chaîne, la conversion retient $\mathrm{Sa} 1=\mathrm{Sé} 2$.

\footnotetext{
${ }^{11}$ Aristote, Poétique, (1457b).
} 
affirmation qui, malgré son caractère obscur, ne laisse pas... l'ombre d'un doute sur la conception de la métaphore mise en jeu.

Bernicot (1981) entreprend une étude de psychologie expérimentale sur les métaphores en présentant plusieurs caractérisations, qu'elle confronte, mais sans remettre en question le dogme "pléthonomique », dogme qu'elle présuppose dans sa partie définitoire (p. 468) : « cependant, contrairement à la métaphore, la comparaison n'impose pas un transfert de signification ».

Bouverot (1969) traite de problèmes difficiles et importants, mais toujours formulés dans le 'langage' du transfert de sens, ce qui ne l'empêche pas, néanmoins, d'aborder des points très originaux susceptibles de remettre en cause, précisément, la distinction propre / figuré. Ainsi, par exemple, p. $234:$ : [...] la substitution métaphorique résulte d'un choix, ce qui légitime les études stylistiques à son propos. Mais il serait faux aussi d'en faire l'apanage des poètes $[\ldots] »$.

Dumarsais (Chesneau Du Marsais 1730 : 125) considère, quant à lui, que : La métaphore est une figure par laquelle on transporte, pour ainsi dire, la signification propre d'un nom à une autre signification qui ne lui convient qu'en vertu d'une comparaison qui est dans l'esprit. Un mot pris dans un sens métaphorique perd sa signification propre et en prend une nouvelle qui ne se présente à l'esprit que par la comparaison que l'on fait entre le sens propre de ce mot et ce qu'on lui compare $[\ldots]$.

ce qui constitue une des formulations les plus précises du point de vue critiqué ici.

Kerbrat-Orecchioni (1994) maintient que

L'identification d'un trope implique donc toujours la reconnaissance d'un décalage entre sens littéral et sens actualisé, donc l'identification conjointe de ces deux niveaux de contenu, ainsi que la possibilité de les hiérarchiser à l'inverse de la normale »

tout en reconnaissant que « ces différentes opérations ne sont pas toujours aisées à réaliser » (p. 59).

Ortony et al. (1978) ne remettent pas en question l'approche naïve de Searle (voir ci-dessous) :

Searle's analysis of the comprehension process for metaphors ${ }^{12}$, therefore, entails three stages. First, the literal meaning of the utterance is determined. Second, that meaning is checked against the context. Third, if there is a conflict between the literal meaning and the context, it is reinterpreted and a conveyed meaning is derived. même si leurs expérimentations tendent à prouver que

whether one target will be comprehended more quickly than another depends, not

12 Voir Searle (1979), cité comme « to appear » dans (Ortony et al. 1978). 
so much on whether it has to be interpreted literally, but upon the extent to which it can be readily interpreted in terms of a reader's or listener's emerging representation of what has gone before.

Searle (1979) développe cette conception de la métaphore fondée sur un questionnement qu'il formule de la manière suivante :

The existence of such utterances - utterances in which the speaker means metaphorically something different from what the sentence means literally - poses a series of questions for any theory of language and communication: What is metaphor, and how does it differ from both literal and other forms of figurative utterances? Why do we use expressions metaphorically instead of saying exactly and literally what we mean?

Il est inutile de poursuivre cette fastidieuse énumération ${ }^{13}$ de textes attestant de ce que la conception pléthonomique de la métaphore est extrêmement répandue ; il était néanmoins nécessaire de l'entreprendre pour montrer que la critique de cette conception ne vise pas une position qui ne serait (plus) celle de personne ${ }^{14} \ldots$

Toutes ces conceptions posent explicitement ou présupposent une distinction entre un 'sens propre' et un 'sens figuré' qui en dériverait, et qui seraient tels que les métaphores permettraient de modifier le premier pour obtenir le second. Ce qui permet de différencier les positions de ces auteurs, c'est, d'une part la manière dont ils conçoivent la dérivation de l'un à l'autre, et, d'autre part, leur position sur l'éventuelle rémanence du 'sens propre' dans le 'sens figuré'. En fait, le dogme pléthonomique sert de point de départ à la plupart des travaux sur la métaphore, qu'il soit présenté comme acceptable ou qu'il soit critiqué $^{15}$. Dans sa synthèse de 1975, Ricœur critique parfois la conception de la métaphore comme changement de sens, mais pas pour les raisons linguistiques que nous développons dans cet article ; la position qu'il défend dans (1965 : 405) à propos du potentiel indéfini des signes permet de supposer qu'il souscrirait à la critique linguistique que nous faisons de ce credo.

Par une commodité justifiée par l'abondante littérature, je qualifierai parfois de « conception classique » cette conception pléthonomique de la métaphore comme changement de sens; je montrerai d'abord, non pas qu'il n'existe pas d'effet métaphorique, mais que cette conception classique de l'effet métaphorique est grossièrement erronée. Nous n'aborderons pas ici des sujets qui

${ }^{13}$ Le lecteur qui souhaiterait la prolonger peut parcourir, entre autres, des textes de Cohen, le Groupe MU, Katz, Lebovici, Lallemand, Le Ny, Loffler-Laurian, Nietzsche, Passeron, Prandi, Schifko, Todorov, ... figée...).

${ }^{14} \mathrm{Qu}$ 'elle n'enfonce pas des portes ouvertes (pour broder autour d'une métaphore

${ }^{15}$ Voir, par exemple, Chanay (2002). 
ont été amplement discutés dans cette abondante littérature, non pas par manque d'intérêt, mais, simplement, parce qu'ils ne relèvent pas de notre propos actuel : ainsi en va-t-il de la question de savoir s'il y a une analogie ou une ressemblance qui permet la métaphore, ou si, au contraire, c'est le 'succès' de la métaphore qui fonde l'analogie ou la ressemblance qui lui est associée ; et de même pour la question de la nécessité (ou non) d'une déviance sémantique dans le fonctionnement de la métaphore ${ }^{16}$. On notera que Le Guern (1973), sans remettre véritablement en question le dogme pléthonomique ( L'interprétation de la métaphore n'est possible que grâce au rejet du sens propre », p. 16), l'affaiblit néanmoins suffisamment pour éviter le paradoxe que nous allons souligner dans cette section, et permettre de percevoir les rapports entre métaphore et humour :

« Ainsi Voltaire fait dire au disciple de Leibnitz, dans le dernier chapitre de Micromégas : "mon âme est le miroir de l'univers, et mon corps est la bordure du miroir". La première métaphore prise isolément n'a rien de ridicule : "mon âme est le miroir de l'univers" se comprend facilement grâce à la possibilité que l'on a d'éliminer l'élément de signification "objet matériel" contenu dans le lexème miroir. La seconde métaphore produit un effet comique parce qu'elle ne peut être comprise que si l'on rend au mot "miroir" le sème éliminé dans la première par suite de son incompatibilité avec le contexte. Tout le comique de la phrase vient de ce que l'enchaînement apparemment logique des deux métaphores est inconciliable avec la logique du processus métaphorique. Il est produit par le fonctionnement du langage lui-même, puisque la nature des réalités désignées n'a rien qui puisse susciter le rire ou le sourire » (p. 16 aussi).

Le dogme pléthonomique classique est tellement ancré dans la culture (ou l'idéologie) de notre époque que de nombreux linguistes (?) en sont même arrivés à proposer, comme tâche auxiliaire de la sémantique, la description des processus qui font passer de ce 'sens propre' à ce 'sens figuré'. Fort heureusement, quelques-uns, comme Cadiot ${ }^{17}$, Schulz $^{18}$, Soublin ${ }^{19}$, Tamba et Veyne ${ }^{20}$,

${ }^{16}$ Voir, à ce sujet, Kleiber (1999).

${ }^{17}$ Voir, par exemple, dans Cadiot (2002 : 42) : «Quand ils sont en usage métaphorique, les mots ne perdent rien de leur signification (au contraire, on l'a vu, ils s'y ressourcent et s'y intensifient), mais ils s'insèrent alors différemment dans les jeux collocatifs, paradigmatiques et autres, des énoncés. Ils ont une autre grammaire»

${ }^{18}$ Parmi les différents travaux de Schulz sur la métaphore, on pourra consulter Schulz $(2002,2004,2005)$. Notre travail ne concerne directement que le deuxième des quatre points qu'elle étudie dans ce dernier document.

${ }^{19}$ Cf. Soublin et Tamine (1979, p. 47) : Une définition n'est donc qu'une configuration particulière parmi celles qui offrent le verbe être, et, du point de vue linguistique, elle ne présente aucune particularité qui permettrait de lui opposer ces pseudo-définitions que constituent les métaphores. [...] C'est donc hors du linguistique qu'il faut chercher le principe de leur distinction ».

${ }^{20}$ Tamba et Veyne (1979) expliquent de manière fort convaincante qu'Aristote « ne

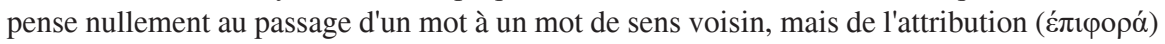


Tamine $^{21}, \ldots$, défendent des positions différentes de cette doxa.

\section{b) Réfutation de la conception classique}

En effet, si l'on admettait sérieusement cette conception 'classique', alors, il s'ensuivrait le paradoxe apparent suivant :

1. Une métaphore modifie le sens de l'énoncé

2. La métaphore est due à la présence d'un mot ou d'un syntagme particulier

3. Ce mot ou ce syntagme n'a plus le sens qui lui permettait de modifier le sens de l'énoncé (puisqu'il a changé de sens, selon la conception 'classique')

4. (= négation de 1) Une métaphore ne modifie pas le sens de l'énoncé

Une illustration, au moyen d'une métaphore nominale simple ${ }^{22}$, permettra de mieux se rendre compte de la portée de cette erreur (qui, rappelons-le, est enseignée presque partout). Considérons un énoncé de la phrase

(1) Max est un loup et demandons-nous : «À quoi peut servir un tel énoncé ?»

Parmi les réponses possibles, on retiendra les trois suivantes :

(i) À dire que « Max » est le nom d'un canidé particulier

(ii) À dire que notre ami Max n'est pas un être humain, mais un canidé

(iii) À suggérer que notre ami Max ressemble à un loup ou se comporte comme un loup dans certaines circonstances

Attirons incidemment l'attention sur la remarque suivante : le fait qu'il soit peu probable qu'un locuteur souhaite accomplir l'action évoquée en (i) et surtout en (ii), ne relève pas de l'étude de la langue française. Dans l'hypothèse

d'un nom à une réalité » (p. 79) ; il y a donc « transfert de dénomination, c'est-à-dire applica-

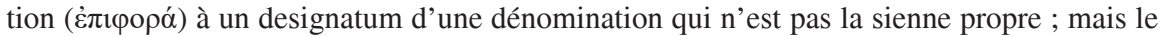
mot ne change pas de sens pour autant $\gg$ (p. 80).

${ }^{21}$ Tamine (1979), tout en reprenant la terminologie propre / figuré montre d'emblée que « quel que soit le cadre de métaphore in praesentia considéré, aucun ne se caractérise par des particularités permettant de distinguer du sens propre le sens figuré » (p.71). Dans sa conclusion (p. 80) l'auteure revient sur ce point en développant l'ide selon laquelle, en ce qui concerne la métaphore, « propre et figuré partagent les mêmes configurations et ne se distinguent que par des critères extérieurs à la linguistique ».

${ }^{22}$ Si l'objectif de cet article était une étude approfondie de la métaphore, nous ne pourrions pas nous contenter de cette illustration : mais il s'agit uniquement de voir pourquoi l'adoption du dogme introduit un paradoxe. L'alternative que nous proposerons ne sera justifiée que pour les cas du type de celui que nous utilisons pour notre illustration : cela est amplement suffisant pour notre objectif, mais nous soutenons, bien entendu, les travaux visant à étendre cette solution aux autres types de métaphores. 
où quelqu'un voudrait accomplir l'action évoquée en (ii), aussi improbable que soit cette hypothèse ou aussi absurde que soit cette action, il pourrait utiliser un énoncé de (1). Le fait que l'objectif (iii), qui oblige à ce que l'on appelle une 'interprétation métaphorique' de (1), soit le plus fréquent n'interdit pas des interprétations 'non métaphoriques' de (1).

Examinons maintenant l'efficacité de l'idée de modification de sens, suggérée par la position 'classique', pour rendre compte de chacun de ces trois usages possibles de (1). Pour les deux premiers usages, il est clair que, si le mot « loup » change de sens et ne renvoie plus à un canidé, on ne peut pas rendre compte des interprétations adéquates. Mais il en va de même pour le troisième usage : si le mot « loup » ne renvoyait plus à l'animal, on ne pourrait pas comprendre à quoi Max ressemble, ou comme quoi il se comporte dans certaines circonstances. Pour obtenir l'effet métaphorique, il est donc nécessaire que le mot « loup » continue à renvoyer au canidé. Ainsi, la conception 'classique' de la métaphore, au lieu d'expliquer l'effet métaphorique, l'interdit au contraire ; le paradoxe apparent auquel elle conduit disparait immédiatement si l'on refuse d'admettre que les métaphores changent le sens des mots. Cette conception 'classique' ne peut donc être admise.

En fait, comme nous allons le voir maintenant, cette conception erronée de la métaphore repose sur une confusion, très répandue, entre le concept d'énoncé et celui de phrase (ou une assimilation partielle des deux concepts).

Cette assimilation erronée entre énoncé (élément de discours) et phrase (unité de langue) conduit, naturellement à une confusion entre unité de discours et unité de langue et, en particulier, à ne pas distinguer les mots-de-discours des mots-de-langue. Elle conduit ainsi à considérer la valeur sémantique d'un énoncé et celle d'une phrase comme deux variétés d'une même espèce, et à confondre sens et signification. Or, si le sens que l'on peut attribuer à un mot-de-discours dépend du sens du discours dans sa situation d'énonciation, la signification d'un mot-de-phrase, elle, ne dépend pas du reste de la phrase : elle est totalement déterminée par les propriétés de la langue dont il s'agit. Ainsi, s'il est légitime de répercuter l'effet métaphorique d'un énoncé sur l'un des mots-de-discours qu'il utilise (par exemple, de répercuter l'effet métaphorique de certains énoncés de (1) sur le mot-de-discours « loup », dans ces énoncés), rien ne permet d'affirmer que ce mot-de-discours (« loup») acquiert son 'sens métaphorique' en raison d'un changement de la signification du mot-de-phrase qui lui est sous-jacent. La croyance que nous critiquons, selon laquelle les métaphores changeraient le sens des mots, ne peut donc pas être comprise comme invoquant un changement de signification des mots-de-phrase, mais seulement comme un changement de sens des mots-de-discours. Mais ce changement étant précisément dû au caractère métaphorique de l'énoncé, il ne peut donc pas en 
être la cause. Le paradoxe apparent qui, on l'a vu, découle de la « conception classique » de la métaphore est dû à ce renversement de causalité, lui-même facilité par le fait de ne pas prendre sérieusement en compte la distinction entre langue et discours.

\subsection{CE QUE LES MÉTAPHORES PEUVENT ÊTRE}

L'intérêt d'une métaphore est, précisément de faire construire un des sens que fait habi-tuellement construire l'interprétation de l'expression clé de la métaphore. Ce n'est qu'en a-doptant une telle conception que l'on peut commencer à comprendre pourquoi les métaphores produisent les effets que nous observons. Dans notre exemple, pour comprendre correctement les différentes possibilités d'interprétation de (1), il est nécessaire d'admettre que l'effet de l'énoncé est d'imposer (ou suggérer) de voir Max comme un loup, dans certaines / toutes cir-constances, et eu égard à certains / tous les aspects de Jean. De manière plus générale, les métaphores imposent (suggèrent) un point de vue sur le thème du discours, à partir duquel l'objectif argumentatif visé est plus à même d'être atteint ${ }^{23}$. Il est fréquent d'interpréter le « voir comme» de Ricœur (1975) comme une simple comparaison : mais dire que X est comme Y est aussi différent de voir X comme Y que dire à quelqu'un « je t'embrasse » ne l'est de l'embrasser... ${ }^{24}$

Un tel objectif argumentatif est, on le voit, compatible avec ce que nous suggérions à la fin de la section 2.4. En fait, imposer/suggérer un point de vue, c'est ce que fait n'importe quel énoncé de n'importe quelle unité de langue. On parle de métaphore lorsque le point de vue suggéré surprend d'une manière ou d'une autre ; quand il cesse de surprendre, le caractère métaphorique se perd. Ainsi, l'impression métaphorique qu'un énoncé est susceptible de pro-duire ne serait rien d'autre qu'une indication du caractère inhabituel des points de vue néces-saires à sa compréhension : tout énoncé serait essentiellement métaphorique dès que sa com-préhension induit des points de vue, et n'apparaîtrait comme tel que lorsque ces points de vue induits paraissent inhabituels.

La notion de métaphore, si elle permet d'évoquer un effet particulier du discours, ne constitue donc pas un principe explicatif en sémantique, puisque ce qui distingue un tel effet de ceux qui sont habituellement produits par les discours (le caractère inhabituel des points de vue nécessaires à la compréhension)

${ }^{23}$ C'est ainsi que l'on peut dire, si l'on ne craint pas de métaphoriser : « dans une métaphore, le phore est le phare du thème »

${ }^{24}$ Un des aspects les plus intéressants de la métaphore est qu'elle permet d'obliger un destinataire à voir Max comme un loup (même pendant un temps très bref), alors que l'énoncé « Max ressemble à un loup » n'oblige absolument pas ses destinataires à le faire. 
ne relève pas des instructions sur la construction du sens, mais d'un jugement sur les points de vue nécessaires à cette construction.

Nous avons ainsi été amenés à considérer que les mécanismes sémantiques sous-jacents aux effets métaphoriques sont les mêmes que les mécanismes sémantiques sous-jacents à ce qu'on appelle habituellement le sens propre, par opposition au sens figuré qui serait 'obtenu par une métaphore'. Ce qui justifie que l'on puisse parler d'effet métaphorique à propos de certains énoncés, c'est que les points de vue que leur compréhension impose sont peu usuels et que ces énoncés les signalent clairement. Lorsqu'on considère qu'un énoncé produit un effet métaphorique, on doit donc lui voir une visée argumentative : celle qui correspond aux points de vue qu'il impose. Il en résulte que tout énoncé clairement métaphorique doit néces-sairement jouer un rôle argumentatif ; cette conséquence n'est pas triviale, puisque tout énoncé ne constitue pas nécessairement une argumentation ${ }^{25}$. L'affirmation (A), selon laquelle tout énoncé clairement métaphorique constitue une argumentation, est réfutable : si elle était fausse, il serait possible de le prouver en trouvant un énoncé clairement métaphorique dont on pourrait prouver qu'il ne constitue pas une argumentation. Comme (A) est une conséquence logique de la proposition que nous avons formulée pour rendre compte de l'effet métaphorique, après l'échec de la 'conception classique', si (A) était fausse, notre proposition devrait être rejetée, malgré ce qu'elle apporte par ailleurs. Il se trouve que (A), bien que réfutable, n'a jamais (pas encore ?) été réfutée : cela ne prouve pas la validité de notre proposition, mais seulement que, jusqu'à présent, on n'a pas pu l'invalider...

Ainsi, tant que l'on n'aura pas réfuté la proposition selon laquelle l'effet métaphorique résulte de l'imposition de points de vue peu habituels à propos de ce dont on parle, que ce soit en réfutant (A) ou par tout autre moyen ${ }^{26}$, les unités de langue permettant aux discours qui les utilisent de produire un effet métaphorique pourront être décrites comme les autres unités de langue, à condition que le modèle sémantique utilisé indique systématiquement les points de vue qu'elles imposent et ceux qu'elles suggèrent ${ }^{27}$.

${ }^{25}$ Les travaux réalisés dans le cadre de l'Argumentation dans la langue ont montré que toute phrase peut être utilisée pour produire un énoncé argumentatif, ce qui ne remet pas en question la possibilité qu'une phrase ne soit pas nécessairement utilisée pour produire un énoncé argumentatif (par exemple, en réponse à la question « quelle heure est-il ?», la réponse «il est onze heure dix » n'est pas une argumentation, cette dernière phrase pouvant être énoncée dans d'autres situations pour reprocher à quelqu'un d'être en retard : ces énoncés, eux, constitueraient des argumentations).

${ }^{26}$ Dans l'état actuel de nos connaissances, nous pensons qu'une telle réfutation n'aura jamais lieu... mais la précaution est indispensable car ce que nous pensons n'a pas de raison d'influencer ce qui peut se produire!

${ }^{27}$ Un tel modèle est justifié dans Raccah (2002) et développé dans Raccah (2010) 


\section{CONCLUSION SUR LES RAPPORTS ENTRE DESCRIPTION SÉMANTIQUE, HUMOUR ET MÉTAPHORE}

Nous venons de voir que la base sémantique d'une métaphore ne peut pas être un changement de signification du segment introduisant l'effet métaphorique, mais la mise en œuvre, à propos de l'objet du discours, des points de vue originaux évoqués par la signification habituelle dudit segment. La compréhension d'un énoncé métaphorique requiert donc, de la part de l'interlocuteur, une phase 'standard' de construction du sens, pouvant conduire à une difficulté d'ajuster le sens construit aux caractéristiques qu'il attribue habituellement à l'objet de discours. Pour résoudre cette difficulté, l'interlocuteur sera amené, dans une deuxième phase, à restreindre l'application des points de vue évoqués par le segment en question à certains aspects de l'objet de discours (dans notre exemple (1), cela peut être le caractère de Max, sa ténacité, sa méchanceté, son appétit, ...).

Au paragraphe 2.2, nous avions vu que, pour un énoncé humoristique, le processus de construction du sens fait intervenir (au moins) deux phases, le sens construit au terme de la dernière phase ayant au moins un aspect incompatible avec le sens construit à l'issue des phases précédentes. Là encore, cette incompatibilité amène l'interlocuteur à remettre en cause l'interprétation initiale.

C'est là que diffère la description de l'effet humoristique de celle de l'effet métaphorique : là où, pour la seconde, un ajustement est possible (et même nécessaire pour que la métaphore soit comprise), pour la première, il est nécessaire qu'aucun ajustement ne soit possible (et plus la distance est grande et infranchissable, plus l'effet humoristique est fort). Mais cette différence concerne les états cognitifs de l'interlocuteur (la possibilité ou non de trouver un ajustement) et non pas les contraintes que les unités de langue imposent à la construction du sens : pour les effets sémantiques qui leur sont sous-jacents, les deux effets sont donc analogues.

Par ailleurs, nous avons vu qu'un modèle sémantique de l'humour doit être en mesure de rendre compte des attentes que le sens construit génère sur les situations évoquées dans les énoncés : pour ce faire, nous avons vu que la description sémantique devait mettre en évidence les points de vue évoqués par les unités de langue utilisées, ainsi que les contraintes que ces dernières imposent aux autres points de vue. Or, à propos de l'effet métaphorique, nous avons vu que, si le modèle sémantique utilisé indique systématiquement les points de vue que les unités de langue imposent et ceux qu'elles suggèrent, il était possible de décrire, dans le même modèle sémantique, les unités de langue permettant aux discours qui les utilisent de produire un effet métaphorique.

Il semble donc souhaitable $e t$ possible de décrire, dans un modèle unique, l'ensemble des phénomènes sémantiques, en rendant compte des analogies entre 
les instructions que les unités 'ordinaires' des langues donnent pour construire du sens, celles qui suggèrent des métaphores, et le schéma d'instructions qui amène à considérer un énoncé comme humoristique.

\section{RÉFÉRENCES BIBLIOGRAPHIQUES}

ATTARDO, Salvatore (1994). Linguistic theories of humor. Berlin ; New York : Mouton de Gruyter.

BARTHES, Roland (1985). L'aventure sémiologique. Paris, Seuil.

CADIOT, Pierre (2002). « Métaphore prédicative nominale et motifs lexicaux », Langue Française 134 : 38-57.

CHESNEAU DU MARSAIS (1730). Des Tropes ou Des diferens sens dans lesquels on peut prendre un mème mot dans une mème langue : ouvrage utile pour l'intelligence des auteurs... Paris, Vve J.-B. Brocas. Reproduction accessible sur le site Gallica de la BNF : http://gallica.bnf.fr/ark:/12148/bpt6k50576m.

FARAL, Edmond (1924). Les arts poétiques du XIIe et du XIIIe siècle. Recherches et documents sur la technique littéraire du moyen âge. Paris, Champion.

GUERN (LE), Michel (1973). Sémantique de la métaphore et de la métonymie. Paris : Larousse

KERBRAT-ORECCHIONI Catherine (1994). Rhétorique et pragmatique : les figures revisitées. Langue française 101 : 57-71.

KLEIBER, Georges (1999). Une métaphore qui ronronne n'est pas toujours un chat heureux. In Nanine CHARBONNEL et Georges KLEIBER (ed.) : La métaphore entre philosophie et rhétorique. Paris, PUF.

LAKOFF, George (1970). A Note on Ambiguity and Vagueness. Linguistic Inquiry, 1: 3.

LALA, Marie-Christine (2005). La métaphore et le linguiste, Figures de la psychanalyse $11: 145-161$.

ORTONY, Andrew, Diane L. SCHALLERT, Ralph E. REYNOLDS, and Stephen J. ANTOS (1978). Interpreting metaphors and idioms: Some effects of context on comprehension. Technical Report No. 93, Center for the study of reading, University of Illinois at Urbana-Champaign. Publié dans Journal of Verbal Learning and Verbal Behavior 17/4 : 465-477.

RACCAH, Pierre-Yves (2002). La semántica de los puntos de vista: hacia una teoría científica y empírica de la construcción del sentido. Letras de Hoje, 129 : 45- 71.

(2005). What is an empirical theory of linguistic meaning a theory of? In Zygmunt FRAJZYNGIER et al. (eds.). Diversity and Language Theory Studies in Language Companion Series, John Benjamins. 
(2010). Racines lexicales de l'argumentation : la cristallisation des points de vue dans les mots. Verbum XXXII-1: 119-141.

(2011). Une conception non paradoxale de la métaphore, s'appuyant sur une conception non métaphorique du paradoxe. In Zsuzsa SIMONFFY (ED.). Le paradoxe et ses usages, Tinta Könyvkiadó, Budapest et Éditions Lambert-Lucas, Limoges

RASTIER, François (1995). Communication ou transmission ? Césure, n 8, 1995, pp. 151-195.

REDDY, Michael J (1979). The conduit metaphor - A case of frame conflict in our language about language. In Ortony, Andrew: Metaphor and thought. Cambridge University Press, pp. 284-324.

RICCEUR PAUL (1965). De l'interprétation. Paris, Seuil.

RICEUR, PAUL (1975). La métaphore vive. Paris, Seuil.

SCHULZ, Patricia (2002). Le caractère relatif de la métaphore. Langue française 134 : Nouvelles approches de la métaphore ; 21-37.

(2004). Description critique du concept traditionnel de "métaphore ». Collection Sciences pour la Communication, Vol. 72. Bern, Peter Lang.

SEARLE, John (1979). Metaphor : In Andrew Ortony (ed.), Metaphor and thought. Cambridge University Press, pp. 83-111.

S.E.L.F. [acronyme de Société d'étude de la langue française, animée, notamment, par Roland Barthes, Jean-Claude Chevalier et Nicolas Ruwet] (1966). Séance du 14 novembre 1964 : le classement structural des figures de rhétorique. Le français moderne 34-1 : 78-79.

TAMBA-MECZ, Irène et Paul VEYNE (1979). Metaphora et comparaison selon Aristote. Revue des Études Grecques, 92 (fascicule 436-437) : 77-98.

DE VINSAUF, Geoffroi (entre 1208 et 1214, selon Faral 1924). Poetria nova, traduit du latin en anglais par Margaret Nims, Toronto, Pontifical Institute of Mediaeval Studies, 1967.

VOLOSHINOV Valentin Nikolaevich. (1929). Марксизм и философия языка [Le marxisme et la philosophie du langage], Leningrad : Priboj. $2^{\mathrm{e}}$ édition : 1930. Traduction française, sous le nom de Mikhaïl BAKHTINE (V.N. VOLOCHINOV) : Le marxisme et la philosophie du langage. Essai d'application de la méthode sociologique en linguistique, Paris : Ed. de Minuit, 1977. 